\title{
Background assessment and performance of the COSINE-100 detector
}

\section{Pushparaj Adhikari*i}

Department of Physics and Astronomy, Sejong University, Seoul 05006, South Korea E-mail:

pushpaparticle@gmail.com

The COSINE-100 dark matter search experiment is an array of $\mathrm{NaI}(\mathrm{Tl})$ crystal detectors located in the Yangyang Underground Laboratory (Y2L). To understand measured backgrounds in the $\mathrm{NaI}(\mathrm{Tl})$ crystals we have performed Monte Carlo simulations using the Geant 4 toolkit and developed background models for each crystal that consider contributions from both internal and external sources, including cosmogenic nuclides. The background models are based on comparisons of measured data with Monte Carlo simulations that are guided by a campaign of material assays and are used to evaluate backgrounds and identify their sources. The average background level for the six crystals ( $70 \mathrm{~kg}$ total mass) that are studied is 3.5 counts/day $/ \mathrm{keV} / \mathrm{kg}$ in the $(2-6$ ) keV energy interval. The dominant contributors in this energy region are found to be ${ }^{210} \mathrm{~Pb}$ and ${ }^{3} \mathrm{H}$.

The 39th International Conference on High Energy Physics (ICHEP2018)

4-11 July, 2018

Seoul, Korea

* Speaker.

${ }^{\dagger}$ COSINE-100 Collaborator 


\section{Background modeling}

COSINE-100 is a dark matter search experiment consisting of a $106 \mathrm{~kg}$ array of eight ultrapure $\mathrm{NaI}(\mathrm{Tl})$ crystals[1]. To model the COSINE-100 detector backgrounds, We have simulated background spectra from radioactive sources: full decay chains of ${ }^{238} \mathrm{U},{ }^{232} \mathrm{Th}$, and ${ }^{40} \mathrm{~K}$ from the crystals, 16 PMTs, and the other external sources; bulk and surface ${ }^{210} \mathrm{~Pb}$ from the crystals; surface $210 \mathrm{~Pb}$ from PTFE reflector; ${ }^{125} \mathrm{I},{ }^{121} \mathrm{Te},{ }^{121 m} \mathrm{Te},{ }^{123 m} \mathrm{Te},{ }^{125 m} \mathrm{Te},{ }^{127 m} \mathrm{Te},{ }^{113} \mathrm{Sn},{ }^{109} \mathrm{Cd},{ }^{22} \mathrm{Na}$, and ${ }^{3} \mathrm{H}$ for cosmogenic isotopes, and have fitted them to the data to estimate their unknown contamination levels [2]. The overall simulated background spectra well describe the measured data from the crystals as shown inFig 1 (a). The low energy background spectra are shown in Fig 1 (b) [2].

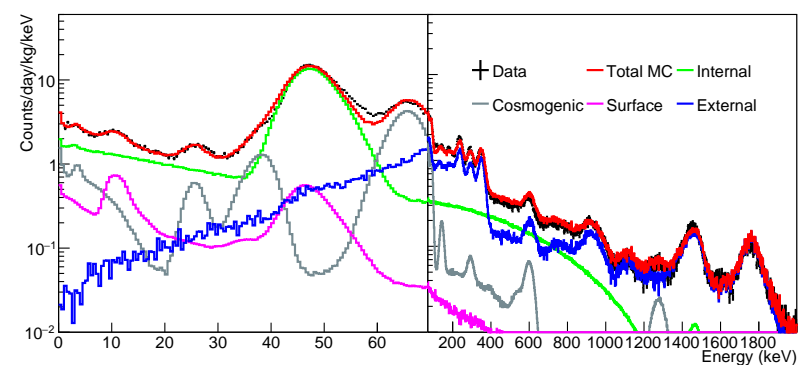

(a)

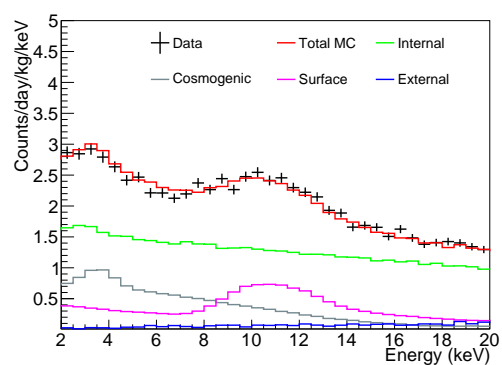

(b)

Figure 1: Comparison of data and Monte Carlo simulation of the single hit background spectra of Crystal-7 in the energy interval $0 \mathrm{keV}-2 \mathrm{MeV}$ (a) and $2 \mathrm{keV}-20 \mathrm{keV}$ (zoom in plot) (b).

\section{Summary}

The simulated background spectra of the $\mathrm{NaI}(\mathrm{Tl})$ detectors well describe the measured data. The background levels in unit of dru (counts $/ \mathrm{day} / \mathrm{keV} / \mathrm{kg}$ ) in the $2-6 \mathrm{keV}$ energy interval is listed in table 1 which shows the dominant background contributions are from ${ }^{210} \mathrm{~Pb}$ and ${ }^{3} \mathrm{H}$ [2].

Table 1: Background events in unit of dru (counts/day/keV/kg) in the $2-6 \mathrm{keV}$ interval.

\begin{tabular}{lccccccc}
\hline Source position & & Crystal-1 & Crystal-2 & Crystal-3 & Crystal-4 & Crystal-6 & Crystal-7 \\
\hline \multirow{3}{*}{ Internal } & ${ }^{40} \mathrm{~K}$ & $0.10 \pm 0.02$ & $0.20 \pm 0.02$ & $0.10 \pm 0.01$ & $0.10 \pm 0.01$ & $0.05 \pm 0.01$ & $0.05 \pm 0.01$ \\
& ${ }^{210} \mathrm{~Pb}$ & $2.50 \pm 0.10$ & $1.69 \pm 0.09$ & $0.57 \pm 0.05$ & $0.71 \pm 0.05$ & $1.46 \pm 0.07$ & $1.50 \pm 0.07$ \\
& Other $\left(\times 10^{-4}\right)$ & $7.0 \pm 0.1$ & $15 \pm 1$ & $7.3 \pm 0.1$ & $7.7 \pm 0.1$ & $14 \pm 1$ & $14 \pm 1$ \\
& & & & & & & \\
Cosmogenic & ${ }^{3} \mathrm{H}$ & $2.35 \pm 0.90$ & $0.81 \pm 0.40$ & $1.54 \pm 0.77$ & $1.97 \pm 0.66$ & $0.69 \pm 0.67$ & $0.58 \pm 0.54$ \\
& ${ }^{109} \mathrm{Cd}$ & $0.05 \pm 0.04$ & $0.009 \pm 0.009$ & $0.13 \pm 0.06$ & $0.29 \pm 0.15$ & $0.08 \pm 0.08$ & $0.09 \pm 0.09$ \\
& $\mathrm{O}$ Other & - & - & $0.02 \pm 0.01$ & $0.09 \pm 0.04$ & $0.06 \pm 0.03$ & $0.05 \pm 0.03$ \\
Surface & & & & & & & \\
External & & $0.64 \pm 0.64$ & $0.51 \pm 0.51$ & $1.16 \pm 0.51$ & $0.22 \pm 0.16$ & $0.34 \pm 0.20$ & $0.38 \pm 0.21$ \\
Total simulation & & $0.03 \pm 0.02$ & $0.05 \pm 0.04$ & $0.03 \pm 0.02$ & $0.03 \pm 0.02$ & $0.04 \pm 0.03$ & $0.03 \pm 0.02$ \\
Data & & $5.68 \pm 1.04$ & $3.28 \pm 0.67$ & $3.57 \pm 0.76$ & $3.41 \pm 0.75$ & $2.74 \pm 0.61$ & $2.70 \pm 0.51$ \\
& & $5.64 \pm 0.10$ & $3.27 \pm 0.07$ & $3.35 \pm 0.07$ & $3.19 \pm 0.05$ & $2.62 \pm 0.05$ & $2.64 \pm 0.05$ \\
\hline
\end{tabular}

\section{References}

[1] G. Adhikari et al. (COSINE-100 Collaboration), Eur. Phys. J. C 78 (2018) 107.

[2] P. Adhikari et al. (COSINE-100 Collaboration), Eur. Phys. J. C 78 (2018) 490. 